\title{
A superconfiguration approach to multi-electron ionization of Xe under strong x-ray irradiation
}

\author{
O Peyrusse $^{1 *}$, B Deschaud ${ }^{1}$ and D Rolles ${ }^{2}$ \\ ${ }^{1}$ Univ. Bordeaux, CEA, CNRS, CELIA (Centre Lasers Intenses et Applications), \\ UMR 5107, F-33400, Talence, France \\ 2 DESY, Notkestraße 85, 22607 Hamburg, Germany
}

\begin{abstract}
The production of highly ionized states in xenon under intense xray irradiation, is discussed with the help of specific calculations. The approach, which retains only one-photon absorption processes (photo-ionization and photoexcitation) as well as Auger and radiative relaxations, makes use of properly defined superconfigurations as global ensemble of configurations. With a tractable number of (super)levels, we explain the occurence of the highest charge states observed in experiments.
\end{abstract}

Submitted to: J. Phys. B: At. Mol. Phys.

(Some figures may appear in colour only in the online journal)

* Email: peyrusse@celia.u-bordeaux1.fr 
A recent study [1] reports the first experimental results on the multiple ionization of heavy atoms (Xe) under intense X-ray irradiation, obtained at a free-electron-laser facility. The highest intensity reached in the experiment was about $10^{17} \mathrm{~W} / \mathrm{cm}^{2}$ while the photon energies were $1.5 \mathrm{keV}$ and $2.0 \mathrm{keV}$, respectively. The target was a very low density xenon gas jet and thus the free electrons produced through photoionization and Auger relaxation were supposed to escape during the interaction. The main diagnostic was a time-of-flight (TOF) spectrometer giving in the ion yield. The striking feature of these results is an unprecendently high degree of ionization of xenon atoms by $1.5 \mathrm{keV}$ XFEL pulses to charge states having ionization energies far exceeding the photon energy. It is of major interest to understand the ionization dynamics of heavy atoms under strong XFEL irradiation because of its implication concerning the electronic damage in high-Z materials and in the context of the diffraction imaging. Accordingly, these studies have been pursued on krypton [2] and also on xenon but with $5.5 \mathrm{keV}$ photons [3].

To simulate the ionization and relaxation dynamics induced by the X-ray pulse, authors of Ref.[1] used a configuration-average description of the numerous atomic states involved, combined with a Monte Carlo method for following the most probable pathways of ionization [4]. While this approach avoids to solve a huge number of coupled equations directly, no resonant excitations to unoccupied bound sub-shells were considered since this would enormously increase the number of configurations. A consequence is that these calculations fail to explain the occurence of high charge states such as $\mathrm{Xe}^{36+}$ especially for $1.5 \mathrm{keV}$ photon irradiation. Authors of Ref.[1] thus invoke the existence of resonance-enabled x-ray multiple ionization (REXMI) pathways that would explain the observation of such high charge states.

Because of the huge number of atomic states involved, the purpose of this communication is to provide an explanation of these data which is based on the use of properly defined superconfigurations allowing to consider the resonant excitations as suggested in Ref. 1. The concept of superconfiguration (SC) which consists in working with supershells defined as a collection of ordinary subshells has been introduced together with the SuperTransition Array (STA) concept in the context of plasma spectroscopy for the purpose of interpreting the high-Z plasma $\mathrm{x}$-ray emission [5]. This technique allows one to consider large ensembles of configurations that need to be taken into account in high-Z plasmas and/or in inner-shell ionized atoms.

It is well established that, in the X-ray regime of interaction, we are far from reaching a non-perturbative regime of interaction (the Keldysh parameter is large compared to unity). A question is, in the perturbative regime, is the multiphoton ionization relevant? It turns out that, for interpreting the data in this regime of interaction ( $\mathrm{keV}$ or multi-keV photons and intensities less than about $10^{17} \mathrm{~W} / \mathrm{cm}^{2}$ ), the simplest scenario consists in retaining only sequential processes (one-photon ionization, one-photon excitation). Note that this simple scenario is less clear for XUV photons [6].

Here, we deliberately focus on the purpose of explaining the occurence of the highest charge states observed in the experiment. We did not try to simulate the exact volume of 
Table 1. Superconfiguration set in neutral Xe with $n_{e x}=3$ (see text).

\begin{tabular}{c} 
superconfiguration \\
\hline$(1 s)^{2}(2 s 2 p)^{8}(3 s 3 p)^{8}(3 d)^{10}(4 s 4 p 4 d)^{18}(5 s 5 p)^{8}$ \\
$(1 s)^{2}(2 s 2 p)^{8}(3 s 3 p)^{8}(3 d)^{9}(4 s 4 p 4 d)^{18}(5 s 5 p)^{8}(4 f 5 d 5 f 6 s 6 p 6 d 6 f 7 s 7 p 7 d 7 f 8 s 8 p 8 d 8 f)$ \\
$(1 s)^{2}(2 s 2 p)^{8}(3 s 3 p)^{7}(3 d)^{10}(4 s 4 p 4 d)^{18}(5 s 5 p)^{8}(4 f 5 d 5 f 6 s 6 p 6 d 6 f 7 s 7 p 7 d 7 f 8 s 8 p 8 d 8 f)$ \\
$(1 s)^{2}(2 s 2 p)^{8}(3 s 3 p)^{8}(3 d)^{8}(4 s 4 p 4 d)^{18}(5 s 5 p)^{8}(4 f 5 d 5 f 6 s 6 p 6 d 6 f 7 s 7 p 7 d 7 f 8 s 8 p 8 d 8 f) 2$ \\
$(1 s)^{2}(2 s 2 p)^{8}(3 s 3 p)^{7}(3 d)^{9}(4 s 4 p 4 d)^{18}(5 s 5 p)^{8}(4 f 5 d 5 f 6 s 6 p 6 d 6 f 7 s 7 p 7 d 7 f 8 s 8 p 8 d 8 f) 2$ \\
$(1 s)^{2}(2 s 2 p)^{8}(3 s 3 p)^{6}(3 d)^{10}(4 s 4 p 4 d)^{18}(5 s 5 p)^{8}(4 f 5 d 5 f 6 s 6 p 6 d 6 f 7 s 7 p 7 d 7 f 8 s 8 p 8 d 8 f) 2$ \\
$(1 s)^{2}(2 s 2 p)^{8}(3 s 3 p)^{8}(3 d)^{7}(4 s 4 p 4 d)^{18}(5 s 5 p)^{8}(4 f 5 d 5 f 6 s 6 p 6 d 6 f 7 s 7 p 7 d 7 f 8 s 8 p 8 d 8 f) 3$ \\
$(1 s)^{2}(2 s 2 p)^{8}(3 s 3 p)^{7}(3 d)^{8}(4 s 4 p 4 d)^{18}(5 s 5 p)^{8}(4 f 5 d 5 f 6 s 6 p 6 d 6 f 7 s 7 p 7 d 7 f 8 s 8 p 8 d 8 f) 3$ \\
$(1 s)^{2}(2 s 2 p)^{8}(3 s 3 p)^{6}(3 d)^{9}(4 s 4 p 4 d)^{18}(5 s 5 p)^{8}(4 f 5 d 5 f 6 s 6 p 6 d 6 f 7 s 7 p 7 d 7 f 8 s 8 p 8 d 8 f) 3$ \\
$(1 s)^{2}(2 s 2 p)^{8}(3 s 3 p)^{5}(3 d)^{10}(4 s 4 p 4 d)^{18}(5 s 5 p)^{8}(4 f 5 d 5 f 6 s 6 p 6 d 6 f 7 s 7 p 7 d 7 f 8 s 8 p 8 d 8 f) 3$ \\
\hline
\end{tabular}

interaction, i.e. the full 3D volume resulting from the interaction of a gaussian intensity beam profile with the gaussian density profile of the gas jet. We just performed zerodimensional (0D) calculations at the highest possible intensity corresponding to the maximum of the intensity profile. In xenon and for $1.5 \mathrm{keV}$ photons, photoionization takes place from the M-shell while photo-excitation can also take place for charge states higher than 18 (28 for $2 \mathrm{keV}$ photons) [1]. For that reason and, exploiting the flexibility of the SC concept, we chose the working superconfigurations to be of the form $(1 s)^{2}(2 s 2 p)^{8}(3 s 3 p)^{n_{3}}(3 d)^{n_{4}}(4 s 4 p 4 d)^{n_{5}}(5 s 5 p)^{n_{6}}(4 f 5 d 5 f 6 s 6 p 6 d 6 f 7 s 7 p 7 d 7 f 8 s 8 p 8 d 8 f)^{n_{7}}$ which, with $n_{3}=8, n_{4}=10, n_{5}=18, n_{6}=8$ and $n_{7}=0$ gives a non-degenerate SC for the ground state of neutral Xe. Varying $n_{7}$ from $0,1,2, \ldots n_{e x}$ (keeping $n_{2}+n_{3}+n_{e x}=18$ in neutral $\mathrm{Xe}$ ) allows one to take into account possible sequential photoexcitations from the $3 s, 3 p, 3 d$ subshells to the supershell ( $4 f 5 d 5 f 6 s 6 p 6 d 6 f 7 s 7 p 7 d 7 f 8 s 8 p 8 d 8 f$ ). For instance, for $n_{e x}=3$, one gets the starting list of superconfigurations as defined in Table 1 for neutral xenon.

From this first list of SCs, one can allow the removal of one electron such that the principal quantum number is greater or equal to 3 (deeper electrons cannot be photoionized by 1.5 or $2 \mathrm{keV}$ photons). This gives another list in $\mathrm{Xe}^{+}$. By removing again one electron (such that the principal quantum number is greater or equal to 3), this new list can be used to generate another list of SCs in $\mathrm{Xe}^{2+}$ and so on. At the end a large list of superlevels is obtained. Its size depends on the chosen value for $n_{e x}$ (from about 20000 for $n_{e x}=0$ to about 95000 for $n_{e x}=5$ ).

In principle, the strict concept of superconfiguration in a plasma involves the notion of internal temperature (which is the electron temperature at local thermodynamical equilibrium). Here this temperature is set to an infinite value which means that our superconfiguration-average quantities do not include Boltzmann factors but just the statistical weights. In that sense, the entity "superconfiguration" is here a pure statistical average. For a whole set of SCs, all x-ray induced processes (photoionization, photoexcitation and stimulated emission) as well as Auger and radiative relaxations, are treated in the same theoretical framework. In other words, all of the couplings 


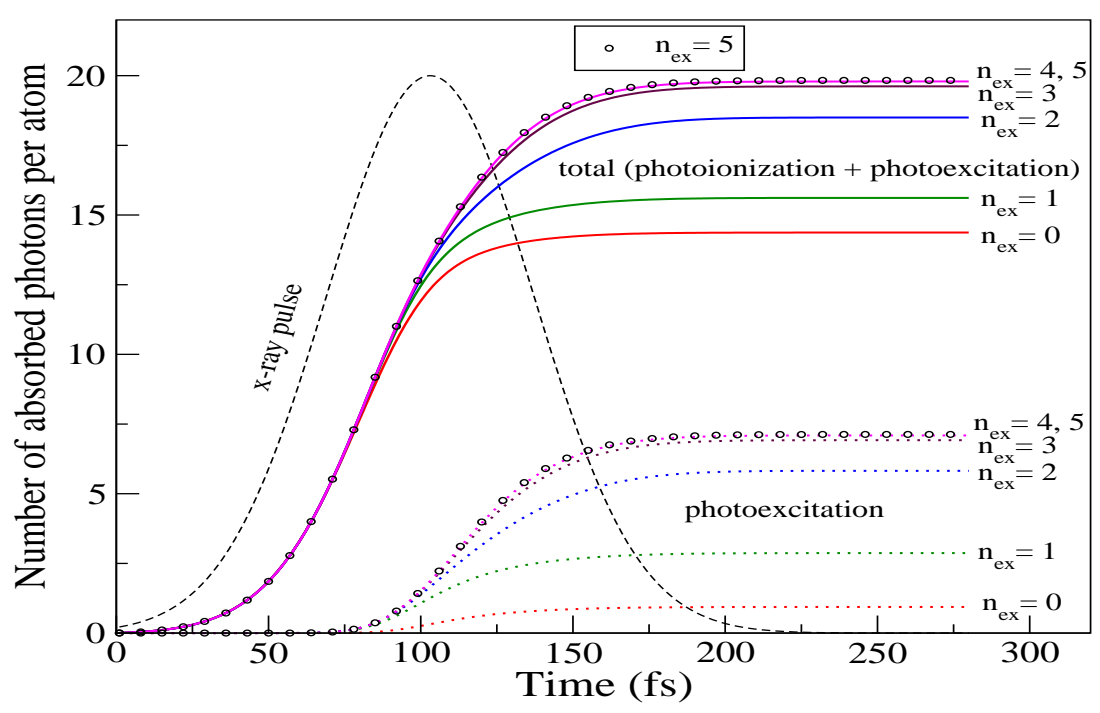

Figure 1. Cumulated average number of photons absorbed per atom as a function of time, by photoionization+photoexcitation and by photoexcitation only, repectively, and for different values of $n_{e x}$ (see text). The intensity is $1.11 \times 10^{17} \mathrm{~W} / \mathrm{cm}^{2}$ and the photon energy is $1.5 \mathrm{keV}$.

between the SCs were calculated by summing over all the possible electron jumps. The corresponding averaging is described in Refs $[7,8]$. At this stage, it should be noted that, each electron jump has its own transition energy which is not the strict difference of energy between the initial and final SCs. Orbital energies were calculated using the Hartree-Fock-Slater (HFS) method. It is worth noting that SC-average quantities are calculated directly [7], i.e. without calculating first configuration-average quantities and performing a subsequent averaging. Also, the radiative coupling between two SCs (of a same charge state) may involve several electron jumps and for each, a spectral profile (of statistical nature) is assigned. Such a profile is called a SuperTransition Array (STA) profile whose position and width must be calculated properly. Furthermore, for the photoionization process, the intrinsic energy spread of the SCs involved and the different possible electron jumps make the threshold not abrupt but defined in the vicinity of the edge as the convolution of a step function and of a gaussian.

In this work, all of the corresponding cross-sections and rates have been precalculated in order to build a set of coupled differential equations governing the populations of the numerous SCs during the pulse. In the present simulations, the XFEL temporal pulse is assumed to have a gaussian temporal envelope with a FWHM of $80 \mathrm{fs}$ and a maximum intensity of $1.11 \times 10^{17} \mathrm{~W} / \mathrm{cm}^{2}$. At each time step, besides the populations, one obtains the total number of photon absorbed per atom. At the end of the pulse, the final population set is used to build a final ion yield.

In order to identify clearly the importance of the so-called REXMI mechanism, we performed various simulations corresponding to different values of $n_{e x}$. To be more specific, we considered the possibility of photo-exciting $n_{e x}=0,1,2, .$. electrons 


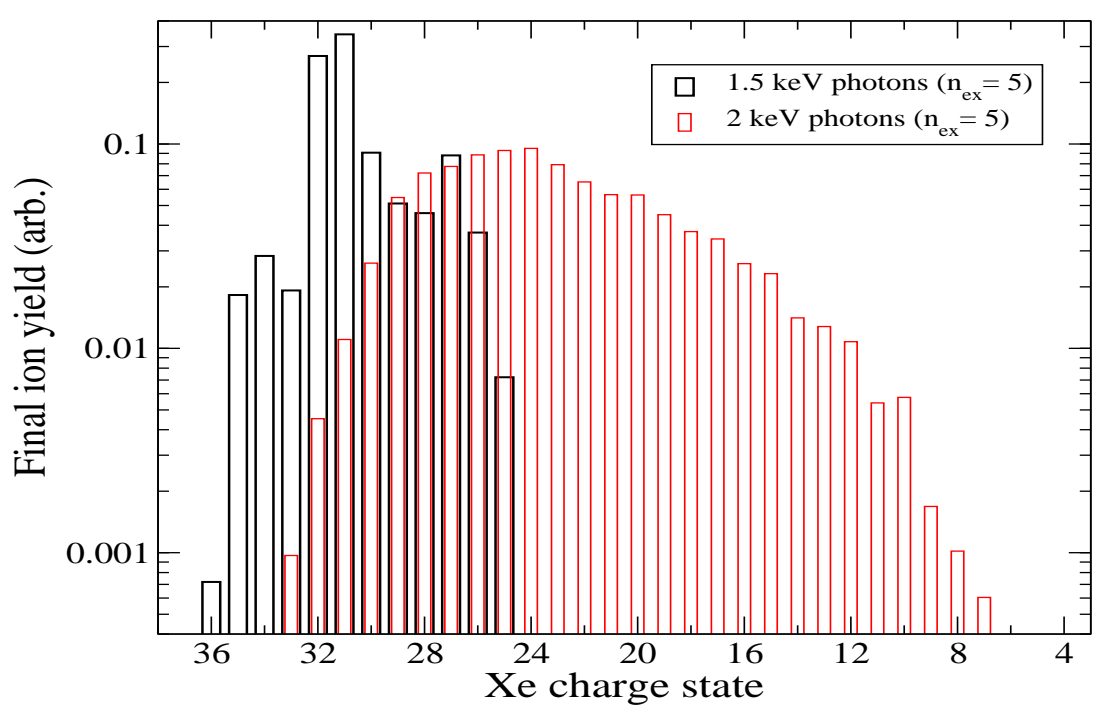

Figure 2. Xenon charge state yields calculated for an 80 fs X-ray pulse, an intensity of $1.11 \times 10^{17} \mathrm{~W} / \mathrm{cm}^{2}$ and photon energies of $1.5 \mathrm{keV}$ and $2.0 \mathrm{keV}$, respectively.

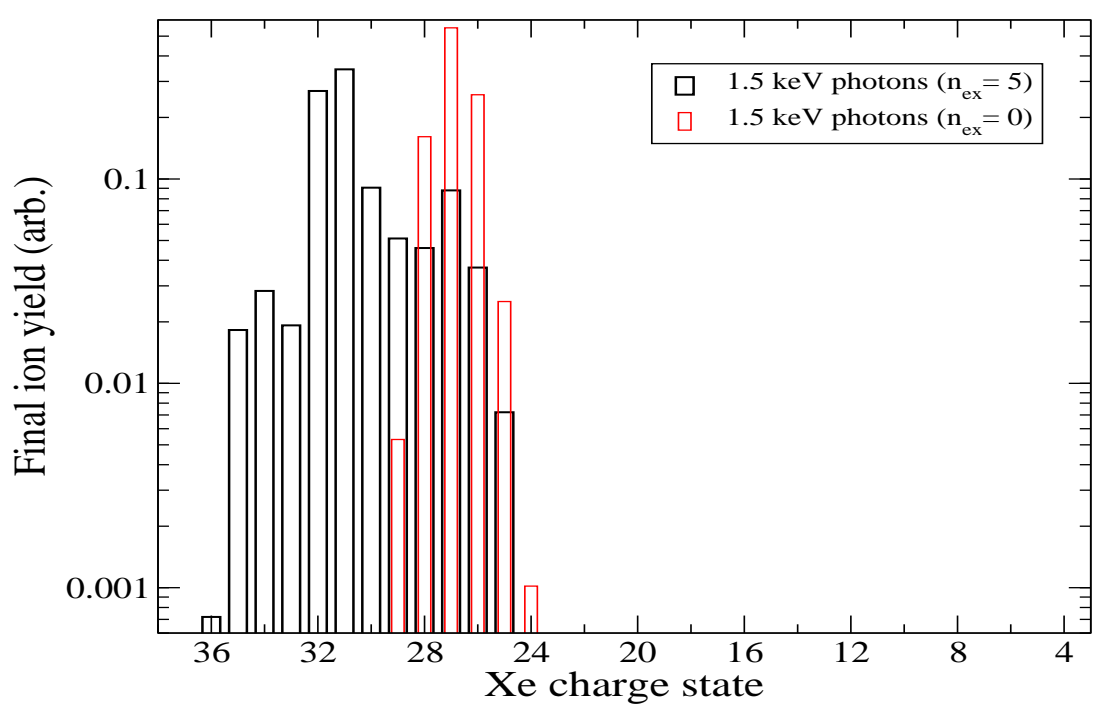

Figure 3. Xenon charge state yields calculated for an $80 \mathrm{fs}$ X-ray pulse, an intensity of $1.11 \times 10^{17} \mathrm{~W} / \mathrm{cm}^{2}$ and a photon energy of $1.5 \mathrm{keV}$. The black bars correspond to a calculation with $n_{e x}=5$ (see text) while the red bars correspond to a calculation with $n_{e x}=0$. 
from the $(3 s, 3 p, 3 d)$ subshells to the $(4 f, 5 d, 5 f, 6 s, 6 p, 6 d, 6 f, 7 s, 7 p, 7 d, 7 f, 8 s, 8 p, 8 d, 8 f)$ subshells. Along with the x-ray pulse shape, the cumulated average number of $1.5 \mathrm{keV}$ photons absorbed (per atom) by photoexcitation and by both mechanisms (photoionization+photoexcitation), respectively, are displayed in figure 1, for different values of $n_{e x}$. One clearly sees that, at some point in the pulse history, photoexcitation processes start to play a role in the ionization dynamics. What is noticeable is a clear convergence of the results for $n_{e x}=4,5$. This convergence obviously depends on the Xray intensity and the converged value of $n_{e x}=5$ is valid only for intensities of the order of $10^{17} \mathrm{~W} / \mathrm{cm}^{2}$. One also notes that the case $n_{e x}=0$ shows a residual photoexcitation due to the vacancies which open in the $(5 s 5 p)$ supershell for ionized Xe.

In figure 2, the final "0D" ion yield calculated with $1.5 \mathrm{keV}$ photons (and $n_{e x}=5$ ) is compared with calculations performed for $2 \mathrm{keV}$ photons. The main point is that, as observed in the experiment [1], the $\mathrm{Xe}^{36+}$ charge state is obtained in the simulation with $1.5 \mathrm{keV}$ photons and not for $2 \mathrm{keV}$ photons (for this energy, resonances can only occur for charge states higher than $\mathrm{Xe}^{28+}$ ). Compared with experimental results, the lowest charge states are less populated in the simulations. There is no doubt that a significant amount of them comes from less irradiated parts of the interaction volume but a full $3 \mathrm{D}$ analyzis is beyond the scope of this communication.

One can also compare the final ion yield obtained for $1.5 \mathrm{keV}$ photons with the largest set of SCs (i.e. the $n_{e x}=5$ case) with the one obtained with the smallest set $\left(n_{e x}=0\right)$ Results are displayed in figure 3 and one clearly sees that one cannot reach the $X e^{36+}$ charge state with a reduced set of SCs that does not include enough excited levels so that photoexcitations occur. Again, this provides evidence of the importance of the highly excited states as relays for further photoionization during the pulse.

To summarize, we corroborate here the suggestion of Ref.[1] concerning the importance of the REXMI mechanism in the ionization dynamics of high-Z materials. Our calculations are based on the use of adequately defined superconfigurations as properly defined ensembles of configurations. This approach allowed us to work with a tractable number (95000) of coupled equations and to consider the photoexcitation mechanism. Despite this somewhat "coarse" aspect of the approach, we have been able to account for the highest charge states observed in the experiment [1] performed at LCLS. It is noteworthy that we considered here only sequential one-photon processes. Of course, present calculations could be refined by splitting somehow the presently defined SCs but at the price of a much less tractable number of coupled equations.

\section{Acknowledgments}

D.R. ackowledges support from the Helmholtz Gemeinschaft through the Young Investigator Program. 


\section{References}

[1] Rudek B, Son S.-K., Rolles D. et al 2012 Nature Photonics 6858

[2] Rudek B, Rolles D., Son S.-K. et al 2013 Phys. Rev. A 87023413

[3] Fukuzawa H. et al 2013 Phys. Rev. Lett. 110173005

[4] Son S.-K. and Santra R. 2012 Phys. Rev. A 85063415

[5] Sorokin A.A. et al 2007 Phys. Rev. Lett. 99213002

[6] Bar-Shalom A. et al 1989 Phys. Rev. A 403183

[7] Peyrusse O 2000 J. Phys. B: At. Mol. Phys. 334303

[8] Peyrusse O 1999 J. Phys. B: At. Mol. Phys. 32683 